ORNL-CTP-95-02

RAL-94-106

hep-ph/9501405

\title{
Hybrid and Conventional Mesons in the Flux Tube Model: Numerical Studies and their Phenomenological Implications
}

\author{
T. Barnes \\ Computational and Theoretical Physics Group, Oak Ridge National Laboratory \\ Oak Ridge, TN 37831-6373, USA \\ and \\ Department of Physics and Astronomy, University of Tennessee \\ Knoxville, TN 37996-1200, USA \\ F.E. Close \\ Daresbury Rutherford Appleton Laboratory \\ Chilton, Didcot, OXON OX11 0QX, England \\ E.S. Swanson \\ Department of Physics, North Carolina State University \\ Raleigh, NC 27695-8202, USA
}

(January 1995)

\begin{abstract}
We present results from analytical and numerical studies of a flux tube model of hybrid mesons. Our numerical results use a Hamiltonian Monte Carlo algorithm and so improve on previous analytical treatments, which assumed small flux tube oscillations and an adiabatic separation of quark and flux tube motion. We find that the small oscillation approximation is inappropriate for typical hadrons and that the hybrid mass is underestimated
\end{abstract}


by the adiabatic approximation. For physical parameters in the "one-bead" flux tube model we estimate the lightest hybrid masses $\left({ }_{\Lambda} L={ }_{1} P\right.$ states $)$ to be 1.8-1.9 GeV for $u \bar{u}$ hybrids, 2.1-2.2 GeV for $s \bar{s}$ and 4.1-4.2 GeV for $c \bar{c}$. We also determine masses of conventional $q \bar{q}$ mesons with $L=0$ to $L=3$ in this model, and confirm good agreement with experimental $J$-averaged multiplet masses. Mass estimates are also given for hybrids with higher orbital and flux-tube excitations. The gap from the lightest hybrid level $\left({ }_{1} P\right)$ to the first hybrid orbital excitation $\left({ }_{1} D\right)$ is predicted to be $\approx 0.4 \mathrm{GeV}$ for light quarks $(q=u, d)$ and $\approx 0.3 \mathrm{GeV}$ for $q=c$. Both ${ }_{1} P$ and ${ }_{1} D$ hybrid multiplets contain the exotics $1^{-+}$and $2^{+-}$; in addition the ${ }_{1} P$ has a $0^{+-}$and the ${ }_{1} D$ contains a $3^{-+}$. Hybrid mesons with doubly-excited flux tubes are also considered. The implications of our results for spectroscopy are discussed, with emphasis on charmonium hybrids, which may be accessible at facilities such as BEPC, KEK, a Tau-Charm Factory, and in $\psi$ production at hadron colliders. 


\section{INTRODUCTION}

The QCD Lagrangian contains quarks and gluons and the successes of perturbative QCD confirm their existence as dynamical degrees of freedom. The behavior of QCD in the strongly interacting low-energy regime, "nonperturbative QCD", is less well understood. Studies using lattice gauge theory have confirmed the presence of confinement and give spectra for conventional mesons and baryons that are in reasonable agreement with experiment [1], but the status of gluonic hadrons in the spectrum has remained obscure.

It is possible that this is now about to change. Candidates for gluonic hadrons have recently been reported which have much in common with theoretical expectations. There are various lattice predictions for the masses of glueballs; the most reliable is presumably for the glueball ground state, which is expected to be a scalar with a mass near 1.5-1.7 GeV [1]. A candidate for the scalar glueball has been reported at $1520 \mathrm{MeV}$ by the Crystal Barrel collaboration at LEAR [2] and may also be evident in central production by NA12/2 [3] at CERN. Possible evidence for a $1^{-+}$light exotic hybrid candidate has been reported in $\rho \pi$ and $f_{2} \pi$ at about $1775 \mathrm{MeV}$ [4 in $\eta \pi$ and especially $\eta^{\prime} \pi$ at $\sim 1.6 \mathrm{GeV}$ by VES [5], and in $f_{1} \pi$ [6] with a resonant phase in the region $1.6-2.2 \mathrm{GeV}$, with production and decay characteristic similar to theoretical expectations for "hybrid" states. A light $1^{-+}$signal in $\eta \pi$ reported by GAMS near $1.4 \mathrm{GeV}$ [7] has been withdrawn, although KEK [8] reports a resonant $1^{-+}$amplitude with a mass and width similar to the $a_{2}(1320)$. Another possibility is that the surprisingly large $\psi^{\prime}$ production at the Tevatron [9] may be due to the formation and decay of metastable hybrid charmonium [10].

In view of the discovery of these candidates for gluonic hadrons it is appropriate to investigate the theoretical models for these states more carefully, to see if the predictions are relatively stable and what level of theoretical uncertainty is present. This paper concentrates on hybrid states, which are formed by combining a gluonic excitation with quarks.

Hybrids have been studied in the literature using the flux tube model [11 17], the MIT bag model [19], an adiabatic heavy-quark bag model [20], constituent gluon models [21,22], 
and heavy-quark lattice gauge theory [23]. In all these approaches the lightest glueball and hybrids ( $H_{q}$, involving $u, d, s$ flavors) are predicted to have masses in the $\approx 1 \frac{1}{2}-2 \mathrm{GeV}$ region. Hybrids are very attractive experimentally since they span complete flavor nonets and are expected to include the lightest $J^{P C}$-exotics (which are forbidden to $q \bar{q}$ ). For recent reviews of hybrids see [24].

Detailed predictions for hybrid spectroscopy were first carried out using the MIT bag model and QCD sum rules. The bag model predictions [19] suffer from parameter uncertainties and possibly additional effects such as gluon self-energies, so the absolute mass scale and the scale of multiplet splittings are somewhat problematical. Conclusions of the bag model studies include the existence of a lightest hybrid meson multiplet at $\sim 1.5 \mathrm{GeV}$ and the presence of a $1^{-+} J^{P C}$-exotic state in this multiplet. In the bag model the lowest $q \bar{q} g$ hybrids have negative parity due to the bag boundary conditions, which give the first TE gluon mode $\left(J^{P}=1^{+}\right)$lower energy than TM $\left(J^{P}=1^{-}\right)$. For heavy quarks it is unrealistic to assume a spherical bag, so Hasenfratz, Horgan, Kuti and Richard [20] introduced an adiabatic bag model in which the bag was allowed to deform in the presence of fixed $Q \bar{Q}$ sources. The resulting $E(R)$ was used in the two-body Schrödinger equation to give mass estimates for hybrids. Masses found for the lightest hybrids were $\approx 3.9 \mathrm{GeV}$ for $c \bar{c}$ (taken from their Fig.2) and $10.49 \mathrm{GeV}$ for $b \bar{b}$. The estimated systematic uncertainty for $b \bar{b}$ hybrids was $\pm 0.2 \mathrm{GeV}$.

QCD sum rules have been applied to the study of hybrids, notably the $1^{-+}$and $0^{--}$ exotics, by several collaborations [25 29]. Early results by these collaborations suggested a light $1^{-+}$exotic hybrid with a mass between $\approx 1 \mathrm{GeV}$ and $\approx 1.7 \mathrm{GeV}$. The $0^{--}$exotics were predicted to lie much higher, at 3.1-3.65 GeV. Unfortunately, much of the more recent work is not consistent with these results, although Balitsky, Dyakonov and Yung (1986) continue to support a mass of $M\left(1^{-+}\right) \sim 1.5 \mathrm{GeV}$. Latorre, Pascual and Narison [26] cite higher masses of $\approx 2.1 \mathrm{GeV}$ for the $u, d 1^{-+}$and $\approx 3.8 \mathrm{GeV}$ for the $0^{--}$. Govaerts et al. [27] estimate $\approx 2.5 \mathrm{GeV}$ for the $1^{-+} q \bar{q} g(q=u, d, s)$, and their other exotic hybrid mass estimates are rather higher than previous references. They conclude however that the sum 
rules for exotic hybrids are unstable, so all these results are suspect. For heavy $1^{-+}$hybrids Narison [26] estimates $4.1 \mathrm{GeV}$ for $c \bar{c}$ and $10.6 \mathrm{GeV}$ for $b \bar{b}$. In contrast, Govaerts et al. find $\approx 4.4-5.3 \mathrm{GeV}$ for $c \bar{c}$ and $\approx 10.6-11.2 \mathrm{GeV}$ for $b \bar{b}$, albeit with reservations regarding the stability of these results. Thus, sum rules have reached no clear consensus regarding the masses of hybrids, and recent results suggest rather higher masses than previously thought. Some technical errors in the earlier sum rule calculations have been reported by Govaerts et al. [28]. Sum rule calculations of decay couplings have also been reported; deViron and Govaerts [29] anticipate a strong $\rho \pi$ decay mode for the $I=1,1^{-+}$exotic.

Constituent gluon models for hybrids were introduced by Horn and Mandula [21] and were subsequently developed by Tanimoto, Iddir et al. and Ishida et al. [22]. Since these models assume a diagonal gluon angular momentum $\ell_{g}$ their predictions for quantum numbers differ somewhat from the other models. For the lightest hybrid states (with $\ell_{g}=0$ ) Horn and Mandula predict nonexotic quantum numbers equivalent to $P$-wave $q \bar{q}$ states, since the gluon has $J^{P}=1^{-}$. Exotic quantum numbers including $1^{-+}$are predicted in the higher-lying $\left(\ell_{q \bar{q}}, \ell_{g}\right)=(1,0)$ and $(0,1)$ multiplets. Detailed spectroscopic predictions for hybrids have not been published using constituent gluon models, and the estimated masses are assigned large uncertainties. A typical result, due to Ishida, Sawazaki, Oda and Yamada, is $1.3-1.8 \mathrm{GeV}$ for light nonexotic hybrids and $1.8-2.2 \mathrm{GeV}$ for light exotics. This type of model predicts that the dominant two-body decay modes of light exotic hybrids such as $1^{-+}$ are the $S+P$ combinations 22 such as $b_{1} \pi$ and $a_{1} \pi$. This conclusion was subsequently supported by studies of the flux tube model.

Lattice QCD will presumably give the most reliable predictions for absolute hybrid masses, although at present this approach has little to say about multiplet splittings. In heavy quark lattice $\mathrm{QCD}$, in which the $Q \bar{Q}$ pair is fixed spatially and the gluonic degrees of freedom are allowed to be excited, the lightest charmonium hybrid was predicted by Michael et al. 232 to have a mass of $m\left(H_{c}\right)_{\text {quenched }}=4.04(3) \mathrm{GeV}$. This reference adds an estimated shift of $0.15 \mathrm{GeV}$ to compensate for the quenched approximation, which leads to a final lattice estimate of $m\left(H_{c}\right)=4.19 \mathrm{GeV}$. Note that a wide range of charm 
quark masses has been assumed in hybrid spectrum calculations; in this HQLGT result a value of $m_{c}=1.32 \mathrm{GeV}$ was used, whereas the flux tube calculations of Isgur, Merlin and Paton [12 14] used $m_{c}=1.77 \mathrm{GeV}$. The sensitivity of the hybrid mass spectrum to $m_{c}$ will be addressed subsequently. The corresponding HQLGT estimates for $b \bar{b}$ hybrids were $m\left(H_{b}\right)_{\text {quenched }}=10.56(3) \mathrm{GeV}$ and $m\left(H_{b}\right)=10.81 \mathrm{GeV}$.

In the flux tube model the more recent calculations [12 14] cite masses of about $1.9 \mathrm{GeV}$ for the lightest $(q=u, d)$ hybrid multiplet, about $4.3 \mathrm{GeV}$ for $c \bar{c}$ hybrids and about 10.8 $\mathrm{GeV}$ for $b \bar{b}$ hybrids. There is an overall variation of about $0.2-0.3 \mathrm{GeV}$ in these predictions, as indicated in Table I. Although multiplet splittings are usually neglected in the flux tube model, a rather large inverted spin-orbit Thomas term was found by Merlin and Paton [14]. The flux tube model also predicts very characteristic two-body decay modes for hybrids [16.17] which have motivated experimental studies of the channels $f_{1} \pi$ and $b_{1} \pi$, and suggest $h_{1} \pi$ and $\rho \pi$ [17] as interesting future possibilities.

The mass predictions for the lowest-lying $\left(1^{-+}\right)$exotic hybrid (which is essentially the mass of the lightest hybrid multiplet) are summarized in Table 1. 
TABLE I. Predicted $1^{-+}$Hybrid Masses.

\begin{tabular}{|c|c|c|c|}
\hline state & mass $(\mathrm{GeV})$ & model & Ref. \\
\hline \multirow[t]{3}{*}{$H_{u, d}$} & $1.3-1.8$ & bag model & 19 \\
\hline & $1.8-2.0$ & flux tube model & $11-14$ \\
\hline & $2.1-2.5$ & QCD sum rules (most after 1984) & 2628 \\
\hline \multirow[t]{4}{*}{$H_{c}$} & $\approx 3.9$ & adiabatic bag model & 20 \\
\hline & $4.2-4.5$ & flux tube model & 1214 \\
\hline & $4.1-5.3$ & QCD sum rules (most after 1984) & 2628 \\
\hline & $4.19(3) \pm$ sys. & HQLGT & 23 \\
\hline \multirow[t]{4}{*}{$H_{b}$} & $10.49(20)$ & adiabatic bag model & 20 \\
\hline & $10.8-11.1$ & flux tube model & 1214 \\
\hline & $10.6-11.2$ & QCD sum rules (most after 1984) & 2628 \\
\hline & $10.81(3) \pm$ sys. & HQLGT & 23 \\
\hline
\end{tabular}


In this paper we carry out improved numerical studies of the flux tube model, which is the most widely cited model for hybrids. Previous flux tube estimates of the hybrid spectrum made several simplifying assumptions, including a small oscillation approximation and an adiabatic separation of quark and flux tube motion [11 15]. In principal these could introduce important systematic biases in the spectrum. We will present numerical results which are free of these approximations, using a Hamiltonian Monte Carlo technique. Since our results for the lightest hybrid masses are quite similar to previous analytical results, we conclude that the approximations made were reasonable, or when they did lead to important numerical inaccuracies (such as in the adiabatic approximation and in the small oscillation approximation at small R) the estimates of corrections to the approximations were sufficiently accurate. Thus, we substantiate previous estimates of hybrid masses in the flux tube model, and we also give masses for higher hybrid excitations using our techniques.

\section{THE FLUX TUBE MODEL}

\section{A. Definitions}

In lattice QCD widely separated static color sources are confined by approximately cylindrical regions of chaotic color fields [31]. The flux tube model is an attempt to describe this phenomenon with a simple dynamical model, and was motivated by the strong coupling expansion of lattice QCD [11] and by early descriptions of flux tubes as cylindrical bags of colored fields [32]. In this model one approximates the confining region between quarks by a string of mass points, "beads", with a confining potential between the beads. Since a line of flux in strong-coupling LGT can be extended only in transverse directions (by the application of plaquette operators), by analogy in the flux tube model one allows only locally transverse spatial fluctuations of the bead positions. For a string of $N$ mass points which connects a quark at site 0 to an antiquark at site $N+1$ we write the flux tube model Hamiltonian as 


$$
\begin{gathered}
H=H_{\text {quarks }}+H_{\text {flux tube }}, \\
H_{\text {quarks }}=-\frac{1}{2 m_{q}} \vec{\nabla}_{q}^{2}-\frac{1}{2 m_{\bar{q}}} \vec{\nabla}_{\bar{q}}^{2}+V_{q \bar{q}}, \\
H_{\text {flux tube }}=-\frac{1}{2 m_{b}} \sum_{i=1}^{N}\left(\sum_{\hat{\eta}_{T}}\left(\hat{\eta}_{T} \cdot \vec{\nabla}_{i}\right)^{2}\right)+\sum_{i=1}^{N+1} V\left(\left|\vec{r}_{i}-\vec{r}_{i-1}\right|\right) .
\end{gathered}
$$

Here $m_{q}$ and $m_{\bar{q}}$ are the quark and antiquark masses, $m_{b}$ is the bead mass, and the $\left\{\hat{\eta}_{T}\right\}$ are two orthogonal unit vectors associated with bead $i$ that are transverse to the local string tangent $\left(\vec{r}_{i+1}-\vec{r}_{i-1}\right) /\left|\vec{r}_{i+1}-\vec{r}_{i-1}\right|$. In this study we use a standard linear form for the string potential,

$$
V\left(\left|\vec{r}_{i}-\vec{r}_{i-1}\right|\right)=a\left|\vec{r}_{i}-\vec{r}_{i-1}\right|
$$

and we usually set the string tension $a$ equal to $1.0 \mathrm{GeV} / \mathrm{fm}$. For our estimates of physical hybrid masses we will augment this with a color Coulomb interaction for $V_{q \bar{q}}$ in (2).

\section{B. Adiabatic Potentials and Flux Tube Parameters}

In the flux tube studies of Isgur, Kokoski, Merlin, and Paton [11] 15] the combined quark and flux tube system is treated using an adiabatic approach as a zeroth order approximation. In the adiabatic analysis one exploits the anticipated fast dynamical response of the flux tube relative to heavy-quark time scales, and separates the flux tube and quark degrees of freedom. This is accomplished by fixing the $q \bar{q}$ separation at $R$ and determining an eigenenergy $E_{\Lambda}(R)$ of the flux tube. Solution of the Schrödinger equation for the $q \bar{q}$ wavefunction in the flux tube ground state potential $E_{0}(R)$ then gives the conventional $q \bar{q}$ meson spectrum in the adiabatic approximation. Hybrids are excited states of the string in this approach, and are found using an excited string potential $E_{\Lambda}(R)$. The lightest hybrid follows from an $E_{1}(R)$ in which the lowest string mode has a single orbital excitation about the $q \bar{q}$ axis.

In previous studies the adiabatic potentials $\left\{E_{\Lambda}(R)\right\}$ were determined assuming small string fluctuations relative to the $q \bar{q}$ axis. We shall find that this is an inaccurate approximation for typical hadrons, assuming $R \approx 1 \mathrm{fm}$. 
One motivation for the small oscillation approximation is that it leads to relatively simple analytical results; when applied to (3) it gives a quadratic Hamiltonian, which can be diagonalized using Fourier modes. To illustrate this, consider a string with fixed ends at $\mathbf{x}_{0}=(0,0,0)$ and $\mathbf{x}_{N+1}=(0,0, R)$ and $N$ dynamical beads, with motion allowed only in the transverse $\left\{x_{i}, y_{i}\right\}$ directions. In the small oscillation approximation, assuming that the beads are equally spaced in $z$ by $a_{0}$, so $z_{n}=n a_{0}$ and $a_{0}=R /(N+1)$, the flux tube Hamiltonian becomes

$$
H_{\text {flux tube }}=a R-\frac{1}{2 m_{b}} \sum_{i=1}^{N}\left(\frac{\partial^{2}}{\partial x_{i}^{2}}+\frac{\partial^{2}}{\partial y_{i}^{2}}\right)+\frac{a / a_{0}}{2} \sum_{i=1}^{N+1}\left(\left(x_{i}-x_{i-1}\right)^{2}+\left(y_{i}-y_{i-1}\right)^{2}\right) .
$$

This is equivalent to a system of $N$ coupled masses $\left\{m_{b}\right\}$ with an effective spring constant of $k=a / a_{0}=(N+1) a / R$. We can diagonalize this using sine variables

$$
s_{n, \lambda=(1,2)}=\sqrt{\frac{2}{N+1}} \sum_{i=1}^{N} \sin \left(k_{n} z_{i}\right)(x, y)_{i}
$$

and

$$
(x, y)_{i}=\sqrt{\frac{2}{N+1}} \sum_{n=1}^{N} \sin \left(k_{n} z_{i}\right) s_{n, \lambda=(1,2)}
$$

where $k_{n}=\pi n / R$. This gives

$$
H_{\text {flux tube }}=a R+\sum_{n=1}^{N} \sum_{\lambda=1}^{2}\left(-\frac{1}{2 m_{b}} \frac{\partial^{2}}{\partial s_{n \lambda}^{2}}+\frac{1}{2} \kappa_{n} s_{n \lambda}^{2}\right)
$$

where the effective spring constant of the $n$th Fourier mode is

$$
\kappa_{n}=\frac{4(N+1) a}{R} \sin ^{2}\left(\frac{\pi n}{2(N+1)}\right) .
$$

The ground state energy of the string, which is used as the adiabatic potential for conventional $(q \bar{q})$ mesons, is $a R$ plus the sum of $\omega / 2$ for each mode in the small oscillation approximation. The individual eigenfrequencies are

$$
\omega_{n}=\sqrt{\kappa_{n} / m_{b}}=2 \sqrt{\frac{(N+1) a}{m_{b} R}} \sin \left(\frac{\pi n}{2(N+1)}\right)
$$

and the mode sum runs over $n=1$ to $N$ and $\lambda=1,2$. The resulting ground state energy is 


$$
E_{0}(R)=a R+\sum_{\text {modes }} \frac{1}{2} \omega_{n}=a R+\sqrt{\frac{2(N+1) a}{m_{b} R}}\left\{\frac{\sin \left(\frac{\pi N}{4(N+1)}\right)}{\sin \left(\frac{\pi}{4(N+1)}\right)}\right\},
$$

which agrees with the result of Isgur and Paton [11]. The most general adiabatic potential in the small oscillation approximation is

$$
E(R)=E_{0}(R)+\sum_{\substack{\text { modes } \\ m}} n_{m} \omega_{m}(R)
$$

where $n_{m}$ is the number of excitations of the $m$ th flux tube mode.

The ground state wavefunction of the string in the small oscillation approximation is a Gaussian in the Fourier mode amplitudes,

$$
\Psi_{0}\left(\left\{x_{i}, y_{i}\right\}\right)=\prod_{n, \lambda} \eta_{n} e^{-s_{n \lambda}^{2} / 2 \sigma_{n}^{2}}
$$

where the Gaussian width of mode $n, \lambda$ is given by

$$
\sigma_{n}=\frac{1}{\sqrt{m_{b} \omega_{n}}}=\frac{\left[\frac{R}{(N+1) a m_{b}}\right]^{1 / 4}}{\left[2 \sin \left(\frac{\pi n}{2(N+1)}\right)\right]^{1 / 2}} .
$$

This suggests an estimate of the range of validity of the small oscillation approximation; it should fail when these fluctuations become comparable to $R$.

Excitations can be created from the ground state wavefunction (13) through the application of "phonon" creation operators

$$
A_{n, \lambda}^{\dagger}=\frac{1}{\sqrt{2 m_{b} \omega_{n}}}\left(-\frac{\partial}{\partial s_{n \lambda}}+m_{b} \omega_{n} s_{n \lambda}\right)
$$

with an increase in energy of $\omega_{n}$. States with definite angular momentum component $\Lambda$ along the $q \bar{q}$-axis, which are useful in constructing hybrid states, are created by the linear combinations

$$
A_{n, \Lambda= \pm 1}^{\dagger}=\frac{1}{\sqrt{2}}\left(\mp A_{n, 1}^{\dagger}-i A_{n, 2}^{\dagger}\right)
$$

The flux tube parameters $a, m_{b}$ and $N$ can be constrained by the plausible requirement that the maximum propagation velocity on the flux tube be $c$. In the large- $N$ limit this implies (from (10)) 


$$
v_{\max } / c \equiv \lim _{k \rightarrow 0} \frac{\partial \omega}{\partial k}=\sqrt{\frac{a a_{0}}{m_{b}}}=1 .
$$

The length $a_{0}$ might reasonably be identified with the transverse flux tube extent of $\approx 0.2$ $0.3 \mathrm{fm}$ found in a lattice Hamiltonian string theory [30] or the $\approx 0.2-0.4 \mathrm{fm}$ estimated in lattice Monte Carlo QCD [31]. For a typical string tension of $a=1.0 \mathrm{GeV} / \mathrm{fm}$ the constraint (17) implies $m_{b} \approx 0.2-0.4 \mathrm{GeV}$. We take $m_{b}=0.2 \mathrm{GeV}$ as our standard value, since the larger transverse extent of $0.4 \mathrm{fm}$ may represent fluctuations of an intrinsically smaller flux tube.

Isgur, Merlin and Paton [11 14] also treat $a_{0}$ as a fundamental length but allow $N$ to vary continuously with $R$, so that $a_{0}=R /(N+1)$ is constant. The large- $R$ hybrid potential gap of

$$
\lim _{R \rightarrow \infty} \omega_{1}(R)=\sqrt{\frac{a}{m_{b}}} \frac{\pi}{\sqrt{(N+1) R}}
$$

then becomes

$$
\lim _{R \rightarrow \infty} \omega_{1}(R)=\sqrt{\frac{a a_{0}}{m_{b}}} \cdot \frac{\pi}{R}=\frac{\pi}{R} .
$$

The final result follows from the constraint (17). An excitation energy of $\pi / R$ was found earlier by Gnädig et al. [32] in their cylindrical bag model of a flux tube.

Of course we cannot vary $N$ continuously in a numerical simulation. In this first numerical study we shall mainly consider the simplest fixed- $N$ case, $N=1$. As we shall see, this allows a detailed study of the various approximations used previously in estimating hybrid masses, and leads to very plausible results for conventional and hybrid spectroscopy.

\section{NUMERICAL RESULTS FOR ADIABATIC POTENTIALS}

We will now generate adiabatic potentials numerically, for comparison with the small oscillation potentials derived in the previous section.

The adiabatic $N=1$ (single bead) problem can be integrated numerically, since there is only motion in a single plane, and the bead wavefunction can be separated as $\Psi_{\Lambda}(\rho, \theta)=$ $\psi_{\Lambda}(\rho) \exp (i \Lambda \theta)$. The ordinary differential equation satisfied by $\psi_{\Lambda}(\rho)$ is 


$$
-\frac{1}{2 m_{b}}\left(\frac{d^{2} \psi_{\Lambda}}{d \rho^{2}}+\frac{1}{\rho} \frac{d \psi_{\Lambda}}{d \rho}\right)+\left(2 a \sqrt{\rho^{2}+R^{2} / 4}+\frac{\Lambda^{2}}{2 m_{b} \rho^{2}}\right) \psi_{\Lambda}=E_{\Lambda}(R) \psi_{\Lambda}
$$

and the exact $q \bar{q}$ meson adiabatic potential $E_{0}(R)$ and first hybrid adiabatic potential $E_{1}(R)$ follow from solving this equation for its lowest eigenvalue with $\Lambda=0$ and $\Lambda=1$ respectively. The potentials $E_{0}(R)$ and $E_{1}(R)$ and the potential gap $E_{1}(R)-E_{0}(R)$ are shown in Figs.1 and 2 for $m_{b}=0.2 \mathrm{GeV}$ and $a=1.0 \mathrm{GeV} / \mathrm{fm}$. In the limit of infinitely massive quarks the adiabatic approximation is exact, the $Q \bar{Q}$ separation approaches zero, and the hybrid mass gap is therefore $E_{1}(0)-E_{0}(0)(=0.829 \mathrm{GeV}$ with these parameters). As $R$ increases the potential gap falls, but asymptotically as $2 \sqrt{a / m_{b} R} \quad((10)$ with $n=1$ and $N=1)$ rather than as the $\pi / R$ of Isgur and Paton, due to our assumption of a fixed- $N$ flux tube. The small oscillation adiabatic potentials and gap from (10-12) are shown as dashed lines in Figs. 1 and 2 ; they are evidently useful only beyond $R \approx 1 \mathrm{fm}$. Since $R \approx 1 \mathrm{fm}$ is a typical light $(u, d, s)$ hadron length scale, the small oscillation approximation is inappropriate for light hadrons. For smaller $R$ the approximate small oscillation adiabatic potentials depart considerably from the true $\left\{E_{\Lambda}(R)\right\}$ (solid lines), and actually diverge as $R \rightarrow 0$.

In the previous section we suggested a condition for applicability of the small oscillation approximation, which is that $R$ should be much larger than the zero-point fluctuations $\sigma_{n}$ in the string ground state. The largest fluctuations are in the $n=1$ mode; taking this case, the mode width for $N=1$ is

$$
\sigma_{1}=\left[\frac{R}{4 m_{b} a}\right]^{1 / 4}
$$

Note the weak parameter dependence of the scale of fluctuations implied by the $1 / 4$ power. The characteristic length $R_{c}$ at which the scale of fluctuations $\sigma_{1}$ equals $R$ is given by

$$
R_{c}(N=1)=\left(4 m_{b} a\right)^{-1 / 3}=0.37 \mathrm{fm}
$$

$R$ should be significantly larger than this for the small oscillation approximation to be useful, which is supported by our Figs. 1 and 2 .

Although this paper is primarily concerned with numerical results for the $N=1$ onebead flux tube model, we can carry out simulations for larger $N$ using a Hamiltonian Monte 
Carlo technique [33]. This method will be discussed in the next section, in which it is applied to the combined dynamical quark and flux-tube system. As a test of the Monte Carlo method we confirmed that the adiabatic potentials $E_{0}(R)$ and $E_{1}(R)$ with $N=1$ are accurately reproduced (Fig.2), and we also show results for the $N=2$ case. The hybrid mass gap apparently falls rapidly with increasing $N$, so it may be difficult to find a realistic description of the spectrum with a fixed- $N$ flux tube model for larger $N$; the excitation energy of a many-bead string is presumably quite low relative to the $N=1$ case, assuming similar $m_{b}$ and $a$. There are also rather subtle complications in the dynamics of the $N>1$ flux tube with fixed ends [37]; the constraint of transverse bead motion implies dependence of energies on the initial conditions, which must then be varied to find the lowest-lying state.

\section{HYBRIDS WITH DYNAMICAL QUARKS}

\section{A. Adiabatic Results}

Thus far we have only considered the adiabatic potentials. Now we shall solve the two-body $q \bar{q}$ Schrödinger equation in the exact adiabatic potentials $\left\{E_{\Lambda}(R)\right\}$, which are determined by numerically integrating (20) for a flux tube with static sources separated by $R$. The flux tube ground state and first excited state potentials $E_{0}(R)$ and $E_{1}(R)$ lead to conventional and the lightest hybrid mesons respectively.

For hybrids there is a centrifugal barrier for the $q \bar{q}$ pair that arises from the matrix element of $\vec{L}_{q}^{2}$ in the full quark-and-flux-tube angular momentum eigenstate. The angular wavefunction of the combined gluon or flux tube and quark system was discussed by Horn and Mandula [21] and subsequently by Hasenfratz et al. [20] and Isgur and Paton [11]. There are discrepancies between these references in the $C$ and $P$ hybrid quantum numbers; this does not affect our conclusions regarding hybrid energies because of degeneracies between

the levels concerned. The latter two references give essentially the same rigid body angular wavefunction for the full system, which is 


$$
\psi_{H}^{(L)} \propto \mathcal{D}_{M \Lambda}^{(L)}(\phi, \theta,-\phi)
$$

(The Hasenfratz et al. wavefunction does not have the final $-\phi$ argument because it uses body-fixed rather than space-fixed coordinates.) This is the amplitude to find the $q \bar{q}$ axis pointing along $(\theta, \phi)$ in a hybrid state with total orbital angular momentum $L$ and $\hat{z}$ projection $M$, and $\Lambda$ is the projection of the flux tube orbital angular momentum along the $q \bar{q}$ axis. $\Lambda=n_{m+}-n_{m-}$, where $n_{m \pm}$ is the number of excitations of the mth flux tube mode, $(+)$ for right-handed and $(-)$ for left-handed, as in (16). Thus for a single flux tube excitation $\Lambda= \pm 1$, for doubly-excited flux tubes $\Lambda=0, \pm 2$, and so forth. Parity implies a degeneracy between $\Lambda= \pm|\Lambda|$ levels, so without loss of generality we assume nonnegative $\Lambda$ in our simulations. The total orbital angular momentum $L$ is constrained to be $L \geq|\Lambda|$.

The wavefunction (23) is not fully diagonal in configuration space; it assumes that the flux tube is in a coherent superposition of orientations about the $q \bar{q}$-axis such that the angular momentum projection $\Lambda$ along the $q \bar{q}$ axis is diagonal. This requires a wavefunction

$$
\psi_{f . t .}^{(\Lambda)}\left(\phi_{b}\right)=\frac{1}{\sqrt{2 \pi}} e^{i \Lambda \phi_{b}}
$$

where $\phi_{b}$ gives the rotation of the flux tube about the $q \bar{q}$-axis relative to a reference configuration. In our Monte Carlo we used basis states which are fully diagonal in coordinate space, so a configuration is defined (for $N=1$ ) by the coordinates $\vec{x}_{q}, \vec{x}_{\bar{q}}, \vec{x}_{b}$, which implicitly determine its orientation relative to a reference configuration and space fixed axes, specified by the $q \bar{q}$-axis angles $\theta, \phi$ and the rigid body rotation angle $\phi_{b}$. This relation is defined by the effect of the rotation operator,

$$
\left|\theta, \phi, \phi_{b}\right\rangle=e^{-i \phi J_{z}} e^{-i \theta J_{y}} e^{+i \phi J_{z}}\left|\hat{z}, \phi_{b}\right\rangle
$$

The angles $\theta$ and $\phi$ are specified trivially by the $q \bar{q}$ axis. The rigid body rotation angle $\phi_{b}$ is rather more complicated, and satisfies

$$
\sin \left(\phi_{b}\right)=\frac{\sin (\phi)\left(x_{b}-x_{q \bar{q} \operatorname{cog}}\right)+\cos (\phi)\left(y_{b}-y_{q \bar{q} \operatorname{cog}}\right)}{\left|\vec{r}_{b}-\vec{r}_{q \bar{q} \operatorname{cog}}\right|},
$$


as may be confirmed from Fig.3, which shows the operations required to reach a general configuration from an unrotated "reference" configuration.

Given the $\phi_{b}$ dependence implicit in the $\Lambda$ states, our $\phi_{b}$-diagonal angular wavefunctions must be of the form

$$
\left\langle\theta, \phi, \phi_{b} \mid L, M \Lambda\right\rangle \propto \mathcal{D}_{M \Lambda}^{(L)}\left(\phi, \theta, \phi_{b}-\phi\right)
$$

which we shall use as the guiding wavefunction for hybrid states in the Monte Carlo simulation.

In their equation (28) Isgur and Paton [11] (see also equation (6) of Merlin and Paton [12]) introduce a simple approximation for the matrix element of $\vec{L}_{q}^{2}$, which neglects a mixing

operator that raises and lowers $\Lambda$. This approximation gives $\left\langle\vec{L}_{q}^{2}\right\rangle \approx L(L+1)-\Lambda^{2}$, which transforms the Schrödinger equation into an ordinary differential equation for the adiabatic $q \bar{q}$ radial wavefunction $\psi_{\Lambda}^{(L)}(r)$,

$$
\begin{gathered}
H_{\text {adia. }}=-\frac{1}{2 \mu}\left(\frac{\partial^{2}}{\partial r^{2}}+\frac{2}{r} \frac{\partial}{\partial r}\right)+\frac{L(L+1)-\Lambda^{2}}{2 \mu r^{2}}+E_{\Lambda}(r) \\
H_{\text {adia. }} \psi_{\Lambda}^{(L)}(r)=M_{H} \psi_{\Lambda}^{(L)}(r) .
\end{gathered}
$$

Isgur and Paton determined the hybrid spectrum by solving this eigenvalue problem, with an additional approximation; they replaced the singular small oscillation adiabatic potentials $E_{\Lambda}(R)$ (12) with approximate forms that were nonsingular at $R=0$. We shall instead use the exact (numerical) adiabatic potentials $\left\{E_{\Lambda}(R)\right\}$ (from $\left.(20)\right)$ in $(28,29)$ above, which gives the true adiabatic result for the spectrum. This will be compared to our Monte Carlo results.

\section{B. Monte Carlo Simulation}

We improve on previous studies of the flux tube model by using the Guided Random Walk (GRW) Hamiltonian Monte Carlo algorithm [33] to solve the full $N=1$ model without adiabatic or small oscillation approximations. The GRW algorithm maps the imaginary 
time Schrödinger equation onto a diffusion problem, which is then solved numerically using weighted random walks in the configuration space of the system. The statistical error is reduced through the use of a guiding wavefunction for importance sampling, which is used to determine stepping probabilities between configurations during the walk. This importance sampling does not bias the energies and matrix elements.

In this algorithm a random walk is generated by stepping in the coordinates which define configuration space. For a $q, \bar{q}$ and $N$-bead system there are $N_{x}=2 N+6$ possible coordinates to increment. Starting from a specified initial configuration of quark, antiquark and bead locations at $\tau=0$, one of the coordinates is chosen at random, and an increment $x \rightarrow x+h_{q}$ (or $h_{b}$ ) is made in that coordinate with probability

$$
P(\text { step })=\frac{1}{2} \frac{\psi_{g}\left(x_{\text {new }}\right)}{\psi_{g}\left(x_{\text {current }}\right)}
$$

If the move is not accepted, a move in the opposite direction is made, $x \rightarrow x-h_{q}$ (or $h_{b}$ ). The step sizes in $h_{b}$ (for bead moves) and $h_{q}$ (for quark or antiquark moves, with $m_{q}$ and $m_{\bar{q}}$ assumed equal) are given by

$$
h_{b}=\sqrt{\frac{N_{x} h_{\tau}}{m_{b}}}
$$

and

$$
h_{q}=\sqrt{\frac{m_{b}}{m_{q}}} h_{b}
$$

where $h_{\tau}$ is a small step size in Euclidean time (relative to inverse energy scales). After each move the Euclidean time is incremented by $h_{\tau}$. Excited states with nodes in the guiding wavefunction $\psi_{g}$ require special consideration; for these cases we test that moves do not cross the nodal surface, and if they do they are rejected and another move is generated. This introduces a bias which vanishes as $h_{\tau} \rightarrow 0$. There is also a bias in excited states if a guiding wavefunction is used which has incorrect nodes.

For the static quark simulations in Sec.III we used a guiding wavefunction which is a Gaussian in the total string length $R_{\text {str }}$, 


$$
\psi_{g}=\exp \left\{-\left(R_{s t r} / \xi\right)^{2}\right\}
$$

and allowed only bead moves. The optimum guidance parameter $\xi$ was estimated numerically by minimization of the statistical error, specifically by minimizing the variance of the weight factor $w(\tau)$ in (35). For $N=1$ and all the $R$ values considered here the optimum value was found to be $\xi \approx 1.5 \mathrm{fm}$.

For the dynamical quark ground state we use as our guiding wavefunction

$$
\psi_{g}^{(0)}=\exp \left\{-\left(R_{s t r} / \xi\right)^{2}-R / \xi_{q \bar{q}}\right\}
$$

This simple generalization of the static quark Gaussian (33) includes a suppression of the wavefunction with increasing interquark separation $R$ for fixed string length $R_{\text {str }}$, as is intuitively expected for heavy quarks. For excited- $L q \bar{q}$ and hybrid states the wavefunction is more complicated, and must incorporate nodes to ensure orthogonality to the ground state (see below).

In the course of a random walk from Euclidean time 0 to $\tau$ we generate a path-dependent weight factor, given by

$$
w(\tau)=\exp \left\{\int_{0}^{\tau}\left(-V+\left[\frac{\nabla_{q}^{2} \psi_{g}+\nabla_{\bar{q}}^{2} \psi_{g}}{2 m_{q}}+\frac{\nabla_{b}^{2} \psi_{g}}{2 m_{b}}\right] \psi_{g}^{-1}\right) d \tau\right\},
$$

where the Laplacians are in the 6 quark and antiquark and $2 N$ (transverse) bead coordinates respectively. The form (35) and the step sizes $h_{b}$ and $h_{q}$ above are chosen so that a histogram of these weights in configuration space $\{x\}$ is proportional to a solution $\psi(\{x\}, \tau)$ of the Euclidean time Schrödinger equation. Actually $w(\tau)$ gives the related function $\left.\psi_{g}(\{x\}) \psi(\{x\}, \tau)\right)$ [34]; this $\psi_{g} \psi$ can also be used to determine the ground state energy, and is generated with a smaller statistical error than $\psi$ itself. The energy is determined from the large- $\tau$ behavior of the weight $w(\tau)$ : At large $\tau$ the walk-averaged weight $<w(\tau)>$ approaches an exponential in $\tau$,

$$
\lim _{\tau \rightarrow \infty}<w(\tau)>=\kappa e^{-E_{0} \tau}\left(1+O\left(e^{-E_{g a p} \tau}\right)\right)
$$

so we may determine $E_{0}$ from measurements of $\langle w\rangle$ at two successive Euclidean times, 


$$
E_{0}=\lim _{\tau_{1}, \tau_{2} \rightarrow \infty} \frac{1}{\left(\tau_{2}-\tau_{1}\right)} \ln \left\{\frac{<w\left(\tau_{1}\right)>}{<w\left(\tau_{2}\right)>}\right\} .
$$

In practice we leave $\tau_{2}-\tau_{1}$ fixed and increase $\tau_{1}$ until the $E_{0}$ estimate has converged to the required accuracy.

If a guiding wavefunction $\psi_{g}$ with nodes is used, we recover the lowest energy eigenvalue for which $\psi=0$ on those nodes. If the nodes are identical to those of an excited state $\psi_{n}$ of the system, we recover the correct $E_{n}$ from (37).

This algorithm gives the true eigenenergy for any guiding wavefunction $\psi_{g}$ with correct nodes, provided that the initial configuration has nonzero amplitude in the ground state. The results become statistically more accurate as the guiding wavefunction is made closer to the true eigenfunction $\psi_{n}$, and one may confirm that the best possible choice is an energy eigenfunction, $\psi_{g}=\psi_{n}$ [34]. In this case the weight factor (35) becomes $w=\exp \left(-E_{n} \tau\right)$ exactly for each walk, so the energy can be determined from a single walk at arbitrary $\tau$. Of course we do not know $\psi_{n}$ in general, so we use a parametrized Ansatz for $\psi_{n}$ as our $\psi_{g}$, and determine the optimum parameters numerically by minimizing the variance of the weight factors $\{w\}$ in a sample of random walks. Given the optimized guiding wavefunction $\psi_{g}$, we then determine $E_{n}$ using (37).

\section{Monte Carlo Results}

For $\alpha_{s}=0$ we generated Monte Carlo energies for quark masses of $m_{q}=0.33,0.5,1.0$, 1.5, 2.5, 5.0 and $10.0 \mathrm{GeV}$, with a string tension of $a=1.0 \mathrm{GeV} / \mathrm{fm}$. The optimized guiding wavefunction parameters in (34) were $\xi=1.5 \mathrm{fm}$ and $\xi_{q \bar{q}}=1.4,1.0,0.7,0.6,0.5,0.4$ and $0.3 \mathrm{fm}$ for the quark masses given above. The Euclidean times used, which were chosen to insure convergence to ground state results to within our statistical errors, were $\tau_{1}=10.0$ $\mathrm{GeV}^{-1}$ and $\tau_{2}=\tau_{1}+1.0 \mathrm{GeV}^{-1}$, and the step size was $h_{\tau}=0.005 \mathrm{GeV}^{-1}$. For energy differences of excited and ground state levels, $E_{n}-E_{0}$, we found adequate convergence with a smaller time of $\tau_{1}=5.0 \mathrm{GeV}^{-1}$. We also generated energies for various other guidance 
and time parameters to confirm the accuracy of these results. The sample size was usually $N_{r w}=8 \times 1024$ walks ( 8 separate runs to generate errors), and we used bootstrap on each of the 8 runs to suppress dependence on the initial configuration. (In a bootstrapped run the final configuration of a walk at $\tau=\tau_{2}$ is used as the initial configuration of the next walk at $\tau=0$.) For hybrids with $m_{q}=0.33$ and $0.5 \mathrm{GeV}$ we used longer runs of $N_{r w}=8 \times 4096$ walks to compensate for the larger statistical errors.

The adiabatic ground state energies (from $(28,29)$ with the potential $E_{0}(R)$ of $(20)$ ) and Monte Carlo results for $N=1$ are summarized in Table 2 for $\alpha_{s}=0, m_{b}=0.2 \mathrm{GeV}$ and $a=1.0 \mathrm{GeV} / \mathrm{fm}$.

TABLE II. Adiabatic and Exact (Monte Carlo) Ground State Energies for $N=1$.

\begin{tabular}{ccc}
\hline \hline$m_{q}(\mathrm{GeV})$ & $E_{0}^{\text {adiabatic }}(\mathrm{GeV})$ & $E_{0}^{\text {MonteCarlo }}-E_{0}^{\text {adiabatic }}(\mathrm{GeV})$ \\
\hline 0.33 & 1.985 & $0.274(4)$ \\
0.50 & 1.868 & $0.231(5)$ \\
1.00 & 1.711 & $0.187(3)$ \\
1.50 & 1.638 & $0.164(3)$ \\
2.50 & 1.563 & $0.148(3)$ \\
5.00 & 1.484 & $0.124(2)$ \\
10.0 & 1.425 & $0.114(3)$ \\
\hline \hline
\end{tabular}


Evidently the adiabatic approximation considerably underestimates the ground state energy, by up to $0.3 \mathrm{GeV}$ for light $(u, d)$ quark systems. The discrepancy falls rather slowly with increasing quark mass, approximately as $m_{q}^{-1 / 4}$.

For excited- $L$ quarkonia we generalize the ground state guiding wavefunction to

$$
\psi_{g}^{(L)}=\psi_{g}^{(0)} \cdot R^{L} \cdot f(\theta, \phi),
$$

where the angular function depends on the direction of the $q \bar{q}$ axis, and was taken to be the real part of $Y_{L M}(\theta, \phi)$. (The algorithm requires a real wavefunction for importance sampling.) The radial factor $R^{L}$ is not essential but is expected to be closer to the true $\psi_{0}^{(L)}$, and its inclusion reduces our statistical errors somewhat.

For hybrid states the amplitude to find the system at $\left(\theta, \phi, \phi_{b}\right)$ is given by $(27)$

$$
\psi_{H}^{(L)}\left(\theta, \phi, \phi_{b}\right) \propto \mathcal{D}_{M \Lambda}^{(L)}\left(\phi, \theta, \phi_{b}-\phi\right)=e^{i \Lambda \phi_{b}} e^{i(M-\Lambda) \phi} d_{M \Lambda}^{(L)}(\theta) .
$$

For our full hybrid guiding wavefunction we multiply the real part of this angular function by a radial wavefunction similar to our ground state $\psi_{g}$,

$$
\begin{gathered}
\psi_{g}^{(H)}=\psi_{g}^{(0)} \cdot \rho_{b} R \cdot f\left(\theta, \phi, \phi_{b}\right) \\
f\left(\theta, \phi, \phi_{b}\right)=d_{M \Lambda}^{(L)}(\theta) \cos \left(\Lambda \phi_{b}+(M-\Lambda) \phi\right) .
\end{gathered}
$$

The product of $\rho_{b}$ (the bead-axis distance) and $R$ was introduced as a simple centrifugal suppression factor.

There is a systematic bias in our results for excited states due to the nodal surfaces specified by the angular wavefunctions $f$; these surfaces are exact only in the limit $m_{q} \rightarrow \infty$. For our high statistics quarkonium simulations we used $M=0$ states for simplicity, since they are $\phi$-independent. We checked for evidence of node bias by comparing the energies found using guiding wavefunctions with different magnetic quantum number $M$, which have different nodal surfaces. The bias in $q \bar{q}$ states was at most about $10 \mathrm{MeV}$, comparable to our statistical errors. For the ${ }_{1} P$ hybrid however we found a significant $M$-dependent 
bias; in Fig.4 we show hybrid energies determined using both $M=0$ and $M=1$ in (41). The largest bias was at the smallest quark mass of $m_{q}=0.33 \mathrm{GeV}$, for which we found $E\left({ }_{1} P, M=1\right)-E\left({ }_{1} P, M=0\right)=52(18) \mathrm{MeV}$. This bias will be discussed in more detail in our treatment of hybrids with physical parameters.

Fig.4 shows the $P$-wave and $D$-wave $q \bar{q}$ levels and the first hybrid level $\left({ }_{\Lambda} L={ }_{1} P\right)$ relative to the ground state energy $E_{0}$, using both the adiabatic approximation (lines) and Monte Carlo (points). Our results show that the adiabatic approximation is more accurate for the energy differences $\left\{E_{n}-E_{0}\right\}$, which are the experimentally observable quantities, than for $E_{0}$ itself. The largest discrepancies between adiabatic and Monte Carlo results are $\approx 100 \mathrm{MeV}$, for the $D$-wave and hybrid states at the lightest quark mass of $0.33 \mathrm{GeV}$. Note that the adiabatic approximation overestimates the excited- $L$ energies but underestimates the hybrid energy. Thus, if we use the adiabatic approximation and fit the experimental D-wave levels, we underestimate the light hybrid mass by $\approx 200 \mathrm{MeV}$.

In their analytical study of the flux tube model, Merlin and Paton [12] also found that postadiabatic corrections reduce the excited- $L$ energies and increase the hybrid energy. They find $(q=u, d) P, D$ and ${ }_{1} P$ hybrid energy shifts which are quite similar in relative strength to our Monte Carlo results; this led Isgur and Paton to revise their adiabatic hybrid mass estimate upwards from $1.67 \mathrm{GeV}$ to $\sim 1.9 \mathrm{GeV}[13]$. The overall scale of adiabatic corrections quoted by Merlin and Paton [12] (see especially their Table 6) is about twice as large as we find numerically, but this may be due to their use of the large- $N$ limit, whereas we have specialized to $N=1$.

\section{Physical Hybrid Masses}

The flux tube results discussed in the previous section are not applicable to real hadrons because they do not include the attractive color Coulomb interaction. Without the Coulomb interaction the flux tube at small $R$ gives an SHO-like adiabatic potential (see $E_{0}(R)$ in

Fig.1), which leads to nearly equal $S-P-D$ splittings in the spectrum of conventional $q \bar{q}$ 
mesons (as in Fig.4). A realistic description of the $S-P-D$ splittings requires the familiar "funnel shaped" potential, in which linear confinement is augmented by a short ranged attraction.

In conventional potential models the Coulomb plus linear form

$$
V_{q \bar{q}}(R)=-\frac{4}{3} \frac{\alpha_{s}}{R}+a R+V_{0}
$$

is most often used, with a string tension of $a \approx 0.9-1.0 \mathrm{GeV} / \mathrm{fm}$ giving the best fit. Perturbative QCD predicts that the effective Coulomb interaction strength $\alpha_{s}$ should run with the scale of momentum of the scattered constituents, provided that we are well above any intrinsic mass scales. For resonance physics this requirement is obviously not satisfied, but there is nonetheless clear evidence for a rapid decrease of $\alpha_{s}$ with increasing quark mass; fits to spectroscopy typically require $\alpha_{s} \approx 0.6-0.7$ for $q=u, d, s, \alpha_{s} \approx 0.3-0.4$ for $q=c$ and $\alpha_{s} \approx 0.2$ for $q=b$.

For our realistic parameter set we assume constituent quark masses of $m_{q}=0.33,0.55$ and $1.5 \mathrm{GeV}$ for $q=u(d), s$ and $c$, and again set the string tension equal to $a=1.0 \mathrm{GeV} / \mathrm{fm}$. In addition we include a color Coulomb and constant potential,

$$
V_{q \bar{q}}=-\frac{4}{3} \frac{\alpha_{s}^{f t}}{R}+V_{0}
$$

in the flux tube quark Hamiltonian (2). The additive constant $V_{0}$ is found to be large and negative in potential models, and in the flux tube model is required in part to cancel the zero-point energies of the beads. The coefficient $-4 / 3$ multiplying $\alpha_{s} / r$ in the color Coulomb interaction merits additional comment. In constituent gluon models of hybrids the $q \bar{q}$ pair would be in a color octet, so the $-4 / 3$ would be replaced by $1 / 6$. In the flux tube model, in which gluonic excitations are presumed nonperturbative in $\alpha_{s}$, it may be more realistic to use $-4 / 3$. This can be motivated by noting that at small $R$ the lowest gluonic excitation is a color singlet $q \bar{q}$ pair (hence $-4 / 3$ ) plus a scalar glueball, rather than a $q \bar{q}$ color octet pair with a diverging $+1 / 6$ color Coulomb interaction [36].

The $\alpha_{s}^{f t}$ in the $N=1$ flux tube $V_{q \bar{q}}$ cannot be compared directly to the Coulomb plus linear $\alpha_{s}$, because the fixed- $N$ flux tube gives an SHO-like confining potential at short 
distances (see $E_{0}(R)$ in Fig.1) in addition to the linear term which dominates at large $R$. Since $\alpha_{s}^{f t}$ in the fixed- $N$ flux tube model must cancel this additional contribution to produce a funnel shaped potential comparable to the standard Coulomb plus linear form, it is larger than the potential model $\alpha_{s}$.

We used multiplet-averaged $E_{S}$ and $E_{P}$ energies as input to fix $\alpha_{s}^{f t}$ and $V_{0}$ in each flavor sector. The numbers used were $E_{P}-E_{S}=0.62 \mathrm{GeV}$ for $q=u, d$ (from $I=1$ ) and $0.45 \mathrm{GeV}$ for $c$. The fitted values of $\alpha_{s}^{f t}$ are 1.3 and 0.72 respectively, each determined to a few per cent accuracy. The $E_{P}-E_{S}$ separation proved to be quite sensitive to the strength of the Coulomb potential. The constant $V_{0}$ was fixed separately for each flavor by using the spin-averaged masses $E_{S}^{(I=1)}=0.63 \mathrm{GeV}$ and $E_{S}^{(c \bar{c})}=3.07 \mathrm{GeV}$ as input. This required $V_{0}^{(I=1)}=-1.71 \mathrm{GeV}$ and $V_{0}^{(c \bar{c})}=-1.17 \mathrm{GeV}$. Since these constant contributions cancel zero-point energies, they are not physically relevant. One might expect them to be roughly flavor independent, however, which can be achieved by increasing $m_{c}$ to $1.8 \mathrm{GeV}$; the effect on the hybrid spectrum will be discussed subsequently. For $s \bar{s}$ we used the $u, d$ parameters and simply increased the quark mass to $m_{s}=0.55 \mathrm{GeV}$.

The Monte Carlo technique was used to determine masses of $q \bar{q}$ and hybrid states up to $L=3$. For $L>0 q \bar{q}$ states we used

$$
f^{(L, M)}(\theta, \phi)=P_{L}^{M}(\cos (\theta)) \cos (M \phi)
$$

in the guiding wavefunction (38) and the high statistics runs used $M=0$. For the hybrids we again used the rigid-body angular wavefunction (41). Tests of node dependence were carried out by varying $M$. The simulations used the same statistics as the $\alpha_{s}=0$ studies of the previous section, although we found that $\tau_{1}=5.0 \mathrm{GeV}^{-1}$ sufficed for convergence of level separations to within the statistical errors. These errors were typically about $\pm 5 \mathrm{MeV}$ for quarkonium states and $\pm 10 \mathrm{MeV}$ for hybrids. The guiding wavefunction parameters used in (34) were $\xi_{q \bar{q}}=3 /\left(2 m_{q} \alpha_{s}^{f t}\right)$ (to give an accurate Coulomb wavefunction for S-waves at short distance), and the flux tube length scale $\xi$ was optimized numerically for each state. For all $q \bar{q}$ and $c \bar{c}$ states we found that $\xi=1.5 \mathrm{fm}$ was nearly optimum. For $q \bar{q}$ hybrids we 
found $\xi=1.8 \mathrm{fm}$ for $\Lambda=1$ and $2.4 \mathrm{fm}$ for $\Lambda=2$. (Note that the higher flux tube excitation requires a larger length scale, as expected.) For $c \bar{c}$ hybrids we found slightly smaller flux tube length scales, $\xi=1.6 \mathrm{fm}$ for $\Lambda=1$ and $2.1 \mathrm{fm}$ for $\Lambda=2$. The quarkonium levels were again independent of $M$ to within our statistical erors, but some bias was evident in the hybrids. This bias decreased with increasing $m_{q}$ and $m_{b}$, as expected. The largest bias was found in the light ${ }_{1} P$ hybrid, for which $E(M=1)-E(M=0)=57(9) \mathrm{MeV}$, similar to our findings for $\alpha_{s}=0$. This fell to $36(7) \mathrm{MeV}$ for charmonium. The corresponding $E(M=2)-E(M=0)$ bias for ${ }_{1} D$ was $24(13) \mathrm{MeV}$ for $u \bar{u}$ and $18(9) \mathrm{MeV}$ for $c \bar{c}$. Measurements with $\pm|M|$ appear to give equivalent results. For this work we average over measurements with all values of $|M|=0$ to $L$; the discrepancies given above imply a systematic uncertainty of about \pm 30 $\mathrm{MeV}$ for the $u, d_{1} P$ hybrid, $\pm 20 \mathrm{MeV}$ for the ${ }_{1} P c \bar{c}$ hybrid, and rather less for the other states. This error could be reduced in future work through incorporation of improved nodal surfaces.

Our numerical results with the standard parameter set $\left(m_{q}, m_{b}, \alpha_{s}^{f t}, a\right)=(0.33 \mathrm{GeV}$, $0.2 \mathrm{GeV}, 1.3,1.0 \mathrm{GeV} / \mathrm{fm})$ are shown in Fig.5. The predicted $D$-wave $q \bar{q}$ mass of $1.66(1) \mathrm{GeV}$ is quite reasonable, given the well-established $D$-wave candidates $\rho_{3}(1690), \omega_{3}(1670)$ and $\pi_{2}(1670)$. The $F$-wave $q \bar{q}$ multiplet is predicted to lie at $2.03(2) \mathrm{GeV}$, in good agreement with the $a_{4}(2040), a_{3}(2050)$ and $f_{4}(2050)$. The lightest hybrid multiplet, which has $\Lambda=1$ and $L=1\left({ }_{\Lambda} L={ }_{1} P\right.$ in our notation $)$, is at $1.90 \mathrm{GeV}$ with these parameters. This is identical to the Isgur-Merlin-Paton prediction of $1.9 \mathrm{GeV}$ [12,13]. Since we are using different versions of the flux tube model this agreement is somewhat fortuitous, although we will show that our result is rather insensitive to parameter variations.

In view of the interest in the experimental hybrid candidate at $1775 \mathrm{MeV}$ [ 1 , which may have exotic $J^{P C}=1^{-+}$but $2^{-+}$and $3^{++}$are also possible, we also determined the mass of the radially-excited $L=2 q \bar{q}$ multiplet, which contains the first $I=12^{-+} q \bar{q}$ level expected above the $\pi_{2}(1670)$. (A $3^{++} q \bar{q}$ state would require $L=3$, and since this multiplet has well established members near $2.05 \mathrm{GeV}$ we do not consider this a plausible $q \bar{q}$ assignment.) For 
the radial simulation we multiplied the $q \bar{q}$ guiding wavefunction $\psi_{g}^{(L)}$ in $(38)$ by $\left|R-R_{0}\right|$, and varied the node radius $R_{0}$ until the energies determined by Monte Carlo in the $R>R_{0}$ and $R<R_{0}$ regions were equal. This required $R_{0}=1.5 \mathrm{fm}$ and gave an energy of $E_{D}^{\prime} \approx 2.3 \mathrm{GeV}$, similar to potential model expectations 38 and far above the $1775 \mathrm{MeV}$ state. This state is thus very unlikely to be a radially-excited D-wave $q \bar{q}$.

We find that the first orbitally excited hybrid multiplet $\left({ }_{1} D\right)$ is at $2.30 \mathrm{GeV}, 400 \mathrm{MeV}$ above the lightest $\left({ }_{1} P\right)$ hybrids. The same numerical result was found earlier by Merlin [15] using the adiabatic approximation. This ${ }_{1} D$ multiplet contains the $J^{P C}$ states $(1,2,3)^{ \pm \mp}$ and $2^{ \pm \pm}$, which includes the exotics $1^{-+}, 2^{+-}$and $3^{-+}$. This level is surprisingly high in mass, since a small orbital excitation gap has been anticipated for hybrids, due to the relatively flat hybrid adiabatic potential found by Michael et al. 223 in heavy-quark lattice gauge theory. We shall see that the orbital excitation gap is somewhat smaller for $c \bar{c}$ hybrids in our model, so there is no serious inconsistency with HQLGT results. If the experimental hybrid candidates near $1.8 \mathrm{GeV}$ [4] and 1.6-2.2 GeV [6] are confirmed, it may be useful to search for members of this ${ }_{1} D$ hybrid multiplet near $2.2 \mathrm{GeV}$ (about $0.4 \mathrm{GeV}$ above ${ }_{1} P$ ). A sequence of hybrids with higher orbital excitation is expected in the flux tube model, although these may be increasingly difficult to observe due to small matrix elements with light $q \bar{q}$ states.

We also determined the mass of the lightest $\Lambda=2$ hybrid multiplet, ${ }_{2} D$. These states are found to be quite high in mass, $\approx 2.75 \mathrm{GeV}$, so they should be irrelevant for light quark spectroscopy in the $2 \mathrm{GeV}$ mass region. Merlin and Paton anticipate a lighter twophonon hybrid multiplet, near $2.2 \mathrm{GeV}$ in the adiabatic approximation. In their level the phonon angular momenta cancel $(\Lambda=0$ "paraphononium"), whereas we have considered $\Lambda=2$ "orthophononium". These $\Lambda=0$ two-phonon states have conventional $q \bar{q}$ quantum numbers, which could complicate their identification.

The sensitivity of hybrid mass predictions to parameter variations is an important issue which has received little attention in previous flux tube studies. To investigate this we 
sequentially increased one parameter of the set $\left(m_{q}, m_{b}, \alpha_{s}^{f t}, a\right)$ by $20 \%$; recall that our standard parameter set $(0.33 \mathrm{GeV}, 0.2 \mathrm{GeV}, 1.3,1.0 \mathrm{GeV} / \mathrm{fm})$ gave $\left(P, D,{ }_{1} P,{ }_{1} D\right)$ masses of $\left([1.25]\right.$ (input),1.66,1.90,2.30) GeV. ( $V_{0}$ is always chosen to give $M_{S}=\left(3 M_{\rho}+M_{\pi}\right) / 4=$ $0.63 \mathrm{GeV}$.) The variations of these masses with parameters (with errors of typically \pm 0.01 $\mathrm{GeV}$ ) were

$$
\Delta\left(M-M_{S}\right)\left(P, D,{ }_{1} P,{ }_{1} D\right)(G e V)= \begin{cases}(-0.01,-0.02,-0.01,-0.02) & \left(\Delta m_{q} / m_{q}=0.2\right), \\ (-0.01,+0.01,-0.05,-0.03) & \left(\Delta m_{b} / m_{b}=0.2\right), \\ (+0.07,+0.08,+0.06,+0.09) & \left(\Delta \alpha_{s}^{f t} / \alpha_{s}^{f t}=0.2\right), \\ (+0.05,+0.11,+0.13,+0.16) & (\Delta a / a=0.2) .\end{cases}
$$

This leads to several conclusions about the importance of parameter uncertainties in our flux tube spectrum. First, the level separations are evidently quite insensitive to variations in quark mass. Second, they are sensitive to changes in $\alpha_{s}^{f t}$ and $a$, but the known $P-S$ and $D-S$ $q \bar{q}$ separations preclude any large changes in these parameters. In any case the hybrid and $D$-wave levels behave similarly under changes in $\alpha_{s}^{f t}$ and $a$, so the predicted hybrid to $D$-wave separation is quite stable. Finally, it is the bead mass that leads to the largest uncertainty. The energies do not depend especially strongly on this parameter, but the hybrid and $q \bar{q}$ energy shifts have opposite signs. (This is more evident in (46) below.). Unfortunately the $q \bar{q}$ masses are quite insensitive to $m_{b}$, so ideally we would use a hybrid mass to determine $m_{b}$. To estimate the range of plausible hybrid masses as we vary $m_{b}$ we consider the range $m_{b}=0.2-0.4 \mathrm{GeV} ; 0.2 \mathrm{GeV}$ is our standard value and $0.4 \mathrm{GeV}$ corresponds to a large flux tube length scale (see discussion in Sec.II.B). Over this range of $m_{b}$ we find the masses (with square brackets as input data)

$$
\left(S, P, D,{ }_{1} P,{ }_{1} D\right)(\mathrm{GeV})= \begin{cases}([0.63],[1.25], 1.66,1.90,2.30) & \left(m_{b}=0.2 \mathrm{GeV}\right) \\ ([0.63], 1.27,1.70,1.78,2.22) & \left(m_{b}=0.4 \mathrm{GeV}\right)\end{cases}
$$

With rounding to $0.1 \mathrm{GeV}$ accuracy this leads to our final estimate of the lightest hybrid mass,

$$
M\left({ }_{1} P\right)=1.8-1.9 \mathrm{GeV}
$$


The first orbitally excited hybrid ${ }_{1} D$ and the first $\Lambda=2$ hybrid ${ }_{2} D$ are expected at about $0.4 \mathrm{GeV}$ and $0.8 \mathrm{GeV}$ above the ${ }_{1} P$ hybrid level respectively.

For $s \bar{s}$ quarkonia and hybrids we simply increased $m_{s}$ to $0.55 \mathrm{GeV}$. The resulting level splittings were very similar to the results for $u, d$ states. Using a $P$-wave $s \bar{s}$ mass of $1.50 \mathrm{GeV}$ as input to fix $V_{0}$, our $s \bar{s}$ results are

$$
\left(S, P, D,{ }_{1} P,{ }_{1} D\right)(\mathrm{GeV})=(0.87,[1.50], 1.88,2.17,2.54) \quad\left(m_{b}=0.2 \mathrm{GeV}\right) .
$$

The only significant changes noted were a decrease in the $D$-wave level (relative to $E_{S}$ ) of $\Delta\left(E_{D}-E_{S}\right)=-0.02 \mathrm{GeV}$ and an increase in the ${ }_{1} P$ level by $0.03 \mathrm{GeV}$. Thus we expect the first $s \bar{s}$ hybrid near $M_{D}(s \bar{s})+0.29 \mathrm{GeV}$, about $50 \mathrm{MeV}$ higher above the $D$-wave level than we found for the corresponding $u, d$ states. The dependence on $m_{b}$ was very similar to that found for $u, d$, so our final result for the first $s \bar{s}$ hybrid level ${ }_{1} P$ was $2.1-2.2 \mathrm{GeV}$.

For charmonium and $c \bar{c}$ hybrids with our standard parameters $m_{c}=1.5 \mathrm{GeV}, m_{b}=0.2$ $\mathrm{GeV}, \alpha_{s}^{f t}=0.72$ and $a=1.0 \mathrm{GeV} /$ fm we predict the following levels:

$$
\left(S, P, D,{ }_{1} P,{ }_{1} D\right)(\mathrm{GeV})=([3.07],[3.52], 3.77,4.21,4.48) \quad\left(m_{b}=0.2 \mathrm{GeV}\right)
$$

These are displayed in Fig.6. Note that the predicted $D$-wave $c \bar{c}$ mass of $3.77 \mathrm{GeV}$ is in good agreement with the experimental $\psi(3770)$. With these parameters we expect the lightest charmonium hybrid at $4.2 \mathrm{GeV}$. The first orbital excitation gap of $c \bar{c}$ hybrids in HQLGT was found to be $0.22 \mathrm{GeV}$ by Michael et al. 23] whereas we estimate $0.27 \mathrm{GeV}$; given the approximations this does not represent a serious discrepancy, although we shall see below that it is a rather stable prediction of this version of the flux tube model.

To test the sensitivity of these results to parameters we again increased each parameter in turn by $+20 \%$, which gives the mass shifts

$$
\Delta\left(M-M_{S}\right)\left(P, D,{ }_{1} P,{ }_{1} D\right)(\mathrm{GeV})= \begin{cases}(+0.02,+0.03,+0.04,+0.04) & \left(\Delta m_{c} / m_{c}=0.2\right), \\ (+0.01,+0.02,-0.05,-0.02) & \left(\Delta m_{b} / m_{b}=0.2\right), \\ (+0.10,+0.13,+0.07,+0.011) & \left(\Delta \alpha_{s}^{f t} / \alpha_{s}^{f t}=0.2\right), \\ (+0.04,+0.06,+0.14,+0.14) & (\Delta a / a=0.2) .\end{cases}
$$


Thus for hybrid charmonium we reach similar conclusions regarding parameter uncertainties. The results are quite insensitive to $m_{c}$; increasing $m_{c}$ from $1.5 \mathrm{GeV}$ to $1.8 \mathrm{GeV}$ only increases the first hybrid mass by $40 \mathrm{MeV}$. Since charm quark masses from $1.25 \mathrm{GeV}$ (HQLGT, 23]) to $1.77 \mathrm{GeV}$ (flux tube, [12 14]) have been used in the hybrid literature, it is reassuring to find that the lightest hybrid mass changes by only about $0.1 \mathrm{GeV}$ over this wide range. As with light quarks we find that $a$ and $\alpha_{s}^{f t}$ strongly affect the hybrid mass spectrum, however these parameters are tightly constrained by the known quarkonium spectrum. The largest uncertainty again comes from $m_{b}$, which is not very well determined by the $c \bar{c}$ spectrum nor by more general theoretical considerations. To test a wide range of possible values we again vary $m_{b}$ over the range $m_{b}=0.2-0.4 \mathrm{GeV}$; with $m_{b}=0.4 \mathrm{GeV}$ we find

$$
\left(S, P, D,{ }_{1} P,{ }_{1} D\right)(\mathrm{GeV})=([3.07], 3.54,3.82,4.08,4.37) \quad\left(m_{b}=0.4 \mathrm{GeV}\right) .
$$

Our final result for the lightest hybrid charmonium mass is thus

$$
M\left({ }_{1} P\right)=4.1-4.2 \mathrm{GeV}
$$

and for charmonium we expect the orbital $\left({ }_{1} D\right)$ and doubly-excited $\left({ }_{2} D\right)$ hybrids about $0.3 \mathrm{GeV}$ and $0.7-0.8 \mathrm{GeV}$ above the ${ }_{1} P$ level respectively.

\section{PHENOMENOLOGICAL IMPLICATIONS}

We have studied the fixed- $N$ version of the flux tube model, principally the $N=1$ case, as a numerically tractable version of this type of hadron model.

The ability to reproduce the spectrum of conventional quarkonia with $N=1$ is of interest in its own right. It suggests that we have a unified picture of both quark and fluxtube excitation spectra, thereby generating some confidence in the predicted hybrid masses. In this final section we summarize implications of these results.

Our studies suggest that the adiabatic approximation, used in previous analyses of hybrid meson masses in the flux tube model, underestimates the hybrid mass scale. Our conclusions 
substantiate previous analytical estimates of corrections to the adiabatic approximation 12,14, and lead to hybrid masses that are $\approx 0.1 \mathrm{GeV}$ above the predictions of quenched heavy-quark lattice QCD, but are consistent with these lattice results given their estimated corrections to the quenched approximation.

In contrast to the light quark sector, in which flavor mixing in non-exotics may be important and the $q \bar{q}$ spectrum itself is rather controversial, in heavy-quark systems the $Q \bar{Q}$ spectroscopy is relatively straightforward and special opportunities ensue for the detection of hybrids. Our results support the expectation that heavy hybrids, $H_{Q}$, appear at masses of

$$
M\left(H_{Q}\right) \approx M_{0}(Q \bar{Q})+1 \mathrm{GeV} .
$$

An important feature in heavy $Q \bar{Q}$ spectroscopy is the existence of narrow states spanning a mass range from $\approx M_{0}(Q \bar{Q})$ through $\approx 1 \mathrm{GeV}$ up to the two-body open-flavor threshold (i.e. $\psi$ to $D \bar{D}$ or $\Upsilon$ to $B \bar{B}$ ). So for charmonium hybrids, for example, one anticipates $H_{c}$ states in the resonance region not far above the open charm threshold of $3.73 \mathrm{GeV}$. In our simulations we actually find the first charmonium hybrids at $M\left(H_{c}\right)=4.1-4.2 \mathrm{GeV}$.

Such a prediction is particularly exciting. Charmonium spectroscopy is rather well understood up to about $3.8 \mathrm{GeV}$, so searches for unusual states should be straightforward near this mass. Since only a few open charm channels occur below $4.3 \mathrm{GeV}$, for a considerable range of hybrid masses one might anticipate rather narrow hybrid resonances. This possibility receives additional support from the flux tube model [16.17, which predicts that the dominant two body decay modes of the lowest lying hybrids are an $L=0$ and $L=1 q \bar{q}$ meson pair. These $S+P$ thresholds are rather high in mass, about $4.3 \mathrm{GeV}$ for $c \bar{c}$ hybrids and $11.0 \mathrm{GeV}$ for $b \bar{b}$ hybrids. The possibility that relatively narrow hybrid charmonium states may exist within this $3.8-4.3 \mathrm{GeV}$ window provides an exciting opportunity for $e^{+} e^{-}$ facilities such as BEPC, KEK and a Tau-Charm Factory. If there are indeed hybrids at these masses, one expects that they should be produced copiously by gluon fragmentation at large momentum transfers, for example at the Tevatron. Detection of the $\psi$ or $\psi(3685)$ as 
a signature of hadronic cascade decays of metastable hybrid charmonia has been discussed in ref [10]. (A double cascade from the $c \bar{c}$ continuum to a hybrid and thence to $c \bar{c}$ was proposed for a Tau-Charm Factory by D.V.Bugg, see ref [39].) In practice the usefulness of cascade decays in hybrid searches will depend on their branching fractions to conventional quarkonia.

Determination of the production and decay characteristics of hybrid states is beyond the scope of this study, but we note in passing that progress in this area has been made recently by analytical modelling of flux tube excitations [17,18]. In these references the decay amplitudes of some recently discovered $1^{-+}, 0^{-+}, 1^{--}$and $2^{-+} u, d$-flavored mesons were found to be in good agreement with the predicted properties of hybrid mesons, so the flux tube model may be a useful guide to strong decay modes as well as masses. Widths of the hybrid charmonia calculated in this model support the suggestion that some of these $c \bar{c}$ hybrids are likely to be narrow.

The production of $1^{--}$charmonium vector hybrids seems especially promising. As the flux tube has an orbital excitation about the $q \bar{q}$ axis, and the $q \bar{q}$ themselves have an effective centrifugal barrier due to the flux tube angular momentum, which suppresses the radial $q \bar{q}$ wavefunction at small $r$, we anticipate that the $e^{+} e^{-}$widths $\Gamma_{e e}\left(V_{c}\right)$ should be significantly smaller than those of the conventional $c \bar{c}$ states $\psi$ and $\psi(3686)$.

In light quark systems this wavefunction suppression is not dramatic (see for example the Particle Data Group summary of $V \rightarrow e^{+} e^{-}$[40 for $L=0$ and $L=2 q \bar{q}$ states following the analyses of refs [41]), so we anticipate a significant light hybrid leptonic width $\Gamma_{e e}\left(\rho_{g}\right)$. The principal difficulty here may lie in distinguishing between light conventional and hybrid vector states unambiguously. The recent analyses of the light vector sector by Donnachie and Kalashnikova [42] actually do support the presence of additional vector states, some of which they suggest may be hybrids.

The recent studies of hybrid decays in the flux tube model [17, [8] may allow tests of these possible light vector hybrids. Since the $q \bar{q}$ pair in $V_{g}$ has $S_{q \bar{q}}=0$, whereas conventional $q \bar{q}$ vector states (either ${ }^{3} S_{1}$ or ${ }^{3} D_{1}$ ) have $S_{q \bar{q}}=1$, there are characteristic selection rules for 
decays that discriminate between these spin-singlet and triplet states. In particular, if the $q \bar{q}$ are in a spin singlet (as in the $V_{g}$ vector hybrid case) then the flux tube decay model forbids decays into final states of two spin singlet mesons.

For $J^{P C}=1^{--}$states this selection rule distinguishes rather clearly between conventional and hybrid vector mesons. It implies that in the decays of a light $\rho_{g}$ hybrid $\rho_{g} \nrightarrow \rightarrow \pi h_{1}$, although $\rho_{g} \rightarrow \pi a_{1}$ is allowed. Analogously, $\omega_{g} \not \rightarrow \pi b_{1}$ for hybrid $1^{--} \omega_{g}$ decays; this is opposite to the case of conventional ${ }^{3} L_{1} q \bar{q}$ mesons, for which the $\pi a_{1}$ channel is suppressed relative to $\pi h_{1}$ or $\pi b_{1}$ 43,44]. The extensive analysis of data in ref [41 revealed the clear presence of a $\rho(1450)$ [40] with a strong $\pi a_{1}$ mode but no evidence for $\pi h_{1}$, in accord with expectations for a hybrid. Furthermore, ref 41 finds an $\omega(1440)$ with no evidence for decays into $\pi b_{1}$, again in conflict with expectations for conventional $q \bar{q}\left({ }^{3} S_{1}\right.$ or $\left.{ }^{3} D_{1}\right)$ states but in accord with predictions for hybrid decays.

The branching fractions reported for the $\rho(1450$ ) [41] (see also [18) suggest that there may be mixing between $\rho_{g}$ and radial $\rho$ basis states in this region. If these hybrid states near $1.5 \mathrm{GeV}$ are confirmed, this mixing may explain the low mass relative to the 1.8-2.0 GeV typical of other hybrid candidates. There may also be significant spin-dependent mass shifts in hybrids that were not incorporated in the present study, which reduce spin-singlet masses (such as $V_{g}$ ) relative to the spin triplet states $\left(0^{-+/-+}, 1^{-+/+-}, 2^{-+/+-}\right)$. To test this possibility, analogous experimental investigations of $1^{--}$hybrid charmonia in $e^{+} e^{-}$would be very useful. In contrast, in $b \bar{b}$ systems the suppressed wavefunction at contact is expected to make $H_{b}$ hybrids essentially absent in $e^{+} e^{-}$annihilation. For this reason the charmonium system may be optimal for hybrid searches; conventional $c \bar{c}$ spectroscopy is reasonably well established, and since the $D$-wave coupling $\Gamma_{e e}(\psi(3770))$ is not negligible, it may be possible to observe a moderately suppressed $V_{c}$ vector hybrid signal in $e^{+} e^{-}$annihilation at a Tau Charm Factory [39]. Diffractive photoproduction of charmonium hybrids, $\gamma^{*} P \rightarrow X P$, may also be possible, for example at HERA.

If the mass of the $V_{c}$ is indeed below or near $4.3 \mathrm{GeV}\left(D^{* *} \bar{D}\right.$ threshold), then hadronic cascades to conventional charmonium states, in particular the $\psi(3097)$ and $\psi(3685)$, may 
be important and could provide a good tag [10]. The E835 experiment at Fermilab may be able to observe production of hybrid charmonium through hadronic cascade decays to $\psi \pi \pi$ and $\psi \eta$.

For hybrids which lie above $D^{* *} \bar{D}$ threshold heavy quark symmetry or detailed decay models may be used to distinguish the spin singlet $H_{c}$ from the spin triplet $\psi$ states through their decay systematics. More detailed theoretical study on this and related questions is now warranted.

To summarize, we find that heavy-quark hybrids in the flux tube model lie below $S+P$ thresholds, and for hybrid charmonium this implies that the lightest states should have rather narrow widths. We anticipate that production by gluon jets may be particularly promising and for this case some quantitative estimates already exist [10] based on the masses found here.

In conclusion, we find the lightest hybrid masses in the flux tube model to be $M\left(H_{u, d}\right)=$ 1.8-1.9 GeV and $M\left(H_{c}\right)=4.1-4.2 \mathrm{GeV}$. These results, combined with recent detailed studies of hybrid decay modes [17, 18], provide a clear set of theoretical predictions for hybrids for comparison with experiment.

\section{ACKNOWLEDGMENTS}

We would like to acknowledge useful discussions or communications with E.S.Ackleh, G.Condo, J.Govaerts, N.Isgur, S.Narison, P.R.Page and J.Paton. This research was sponsored in part by the European Community Human Mobility program EURODAFNE, contract number CHRX-CT92-0026; and the United States Department of Energy under contract DE-AC05-840R21400 with Martin Marietta Energy Systems Inc. at Oak Ridge National Laboratory. 
[1] For recent reviews of spectroscopy from lattice gauge theory see S.R.Sharpe, University of Washington report UW/PT 94-15 (Dec. 1994) and D.Weingarten, Nucl. Phys. B (Proc. Suppl.) 34, 29 (1994). Recent high-statistics lattice glueball results include G.Bali et al., Phys. Lett. B309, 378 (1993), $M\left(0^{++}\right)=1.55(5) \mathrm{GeV}$; H.Chen et al., IBM report IBM-HET-94-1 (contribution of A. Vaccarino to Lattice 93$), M\left(0^{++}\right)=1.740(71) \mathrm{GeV}$ and $M\left(2^{++}\right)=2.359(128)$ GeV. Two-pseudoscalar decay widths are discussed by J. Sexton et al., IBM report IBM-HET94-5 (contribution to Lattice 94).

[2] V.V.Anisovich et al., Phys. Lett. B323, 233 (1994); C.Amsler, in Proceedings of the XXVII Int. Conf. on High Energy Physics (Glasgow, 20-27 July 1994), Zürich report UZH-PH-50/94; see also C.Amsler and F.E.Close, "Evidence for Glueballs", Rutherford Laboratory and CERN report (1995, in preparation).

[3] J.P.Peigneux et al., CERN report CERN-SPSLC 94-22, CERN-P281 (August 1994).

[4] G.Condo et al., Phys. Rev. D43, 2787 (1991); the same state may have been seen earlier by D.Aston et al., Nucl. Phys. B189, 15 (1981).

[5] G.M.Beladidze et al., Phys. Lett. B313, 276 (1993).

[6] J.H.Lee et al., Phys. Lett. B323, 227 (1994).

[7] D.Alde et al., Phys. Lett. B205, 397 (1988).

[8] H.Aoyagi et al., Phys. Lett. B314, 246 (1993).

[9] M. Mangano (CDF Collaboration), in Proceedings of the XXIX Rencontres de Moriond (Méribel, 19-26 March 1994), and in Proceedings of the 27th Int. Conf. on High Energy Physics (Glasgow, 21-27 July 1994).

[10] F.E.Close, Rutherford Laboratory report RAL-94-093, hep-ph/9409203 (Phys. Lett. B, in 
press).

[11] N.Isgur and J.Paton, Phys. Lett. 124B, 247 (1983).

[12] J.Merlin and J.Paton, J. Phys. G11, 439 (1985).

[13] N.Isgur and J.Paton, Phys. Rev. D31, 2910 (1985).

[14] J.Merlin and J.Paton, Phys. Rev. D35, 1668 (1987).

[15] J.Merlin, Oxford University Ph.D. thesis (unpublished); J.Paton, personal communication.

[16] N.Isgur, R.Kokoski and J.Paton, Phys. Rev. Lett. 54, 869 (1985).

[17] F.E.Close and P.R.Page, Rutherford Laboratory report RAL-94-116, hep-ph/9411301

[18] F.E.Close and P.R.Page, Rutherford Laboratory report RAL-94-122, hep-ph/9412301.

[19] T.Barnes, Caltech Ph.D. thesis (1977), unpublished; T.Barnes, Nucl. Phys. B158, 171 (1979); T.Barnes and F.E.Close, Phys. Lett. 116B, 365 (1982); M.Chanowitz and S.R.Sharpe, Nucl. Phys. B222, 211 (1983); T.Barnes, F.E.Close and F.deViron, Nucl. Phys. B224, 241 (1983); M.Flensburg, C.Peterson and L.Sköld, Z. Phys. C22, 293 (1984).

[20] P.Hasenfratz, R.R.Horgan, J.Kuti and J.-M.Richard, Phys. Lett. 95B, 299 (1980).

[21] D.Horn and J.Mandula, Phys. Rev. D17, 898 (1978).

[22] M.Tanimoto, Phys. Lett. 116B, 198 (1982); Phys. Rev. D27, 2648 (1983); A.LeYaouanc, L.Oliver, O.Pène, J.-C.Raynal and S.Ono, Z. Phys. C28, 309 (1985); F.Iddir, A.LeYaouanc, L.Oliver, O.Pène, J.-C.Raynal and S.Ono, Phys. Lett. B205, 564 (1988); S.Ishida, H.Sawazaki, M.Oda and K.Yamada, Phys. Rev. D47, 179 (1992); Prog. Theor. Phys. 82, 119 (1989).

[23] S.Perantonis and C.Michael, Nucl. Phys. B347, 854 (1990), and references cited therein.

[24] See for example T.Barnes, ORNL-CCIP-93-11 / RAL-93-065 and F.E.Close, RAL-93-053, in Proceedings of the Third Workshop on the Tau-Charm Factory (Marbella, Spain, 1-6 June 1993); T.Barnes, ORNL-CCIP-93-14 / RAL-93-069 in Proceedings of the Conference on Exclu- 
sive Reactions at High Momentum Transfers (Marciana Marina, Elba, Italy, 24-26 June 1993); F.E.Close, Rep. Prog. Phys. 51, 833 (1988); C.Dover, in Proceedings of the Second Biennial Conference on Low Energy Antiproton Physics (Courmayeur, 14-19 Sept. 1992); A.Dzierba, Indiana University report IUHEE-93-2, in Proceedings of the BNL meeting on Future Directions in Particle and Nuclear Physics at Multi-GeV Hadron Facilities (Brookhaven, N.Y. 4-6 March 1993); S.Godfrey, in Proceedings of the BNL Workshop on Glueballs, Hybrids and Exotic Hadrons (AIP, 1989), ed. S.-U. Chung; D.Hertzog, Nucl. Phys. A558, 499c (1993); N.Isgur, CEBAF-TH-92-31, in Proceedings of the XXVI International Conference on High Energy Physics (Dallas, August 1992); G.Karl, Nucl. Phys. A558, 113c (1993).

[25] I.I.Balitsky, D.I.Dyakanov and A.V.Yung, Phys. Lett. 112B, 71 (1982); Sov. J. Nucl. Phys. 35, 761 (1982); Z. Phys. C33, 265 (1986).

[26] J.I.Latorre, S.Narison, P.Pascual and R.Tarrach, Phys. Lett. 147B, 169 (1984); J.I.Latorre, P.Pascual and S.Narison, Z. Phys. C34, 347 (1987); S.Narison, "QCD Spectral Sum Rules", Lecture Notes in Physics Vol.26, p.375 (World Scientific, 1989).

[27] J.Govaerts, F.deViron, D.Gusbin and J.Weyers, Phys. Lett. 128B, 262 (1983); (E) Phys. Lett. 136B, 445 (1983); J.Govaerts, L.J.Reinders, H.R.Rubinstein and J.Weyers, Nucl. Phys. B258, 215 (1985); J.Govaerts, L.J.Reinders and J.Weyers, Nucl. Phys. B262, 575 (1985); J.Govaerts, L.J.Reinders, P.Francken, X.Gonze and J.Weyers, Nucl. Phys. B284, 674 (1987).

[28] J.Govaerts, F.deViron, D.Gusbin and J.Weyers, Nucl. Phys. B248, 1 (1984).

[29] F.deViron and J.Govaerts, Phys. Rev. Lett. 53, 2207 (1984).

[30] J.H.Merlin and J.Paton, Phys. Rev. D36, 902 (1987).

[31] See for example J.Wosiek and R.W.Haymaker, Phys. Rev. D36, 3297 (1987); A. DiGiacomo, M.Maggiore and S.Olejnik, Phys. Lett. 236B, 199 (1990); H.D.Trottier and R.M.Woloshyn, Phys. Rev. D48, 2290 (1993).

[32] P.Gnädig, P.Hasenfratz, J.Kuti and A.S.Szalay, Phys. Lett. 64B, 62 (1976). 
[33] T.Barnes, G.J.Daniell and D.Storey, Nucl. Phys. B265 [FS15] (1986) 253. Since we calculate only energies in this flux tube simulation, we can reduce statistical errors by using only the "diagonal weight", as discussed in [34].

[34] T.Barnes and D.Kotchan, Phys. Rev. D35, 1947 (1987).

[35] T.Barnes and F.E.Close, Phys. Lett. 123B, 89 (1983).

[36] N.Isgur, personal communication.

[37] T.Barnes and E.S.Swanson (in preparation).

[38] In a nonrelativistic Coulomb plus linear potential model with $\alpha_{s}=0.6, a=0.9 \mathrm{GeV} / \mathrm{fm}$, $m_{q}=0.33 \mathrm{GeV}$, and again using the spin-averaged $M(\rho, \pi)=0.63 \mathrm{GeV}$ to fix $V_{0}=-0.82$ $\mathrm{GeV}$, we find $M(D)=1.66 \mathrm{GeV}$ and $M\left(D^{\prime}\right)=2.31 \mathrm{GeV}$, and a node in the D' wavefunction at $R_{0}=1.47 \mathrm{fm}$. S.Godfrey and N.Isgur, Phys. Rev. D32, 189 (1985), quote a much lower ${ }^{1} D_{2}^{\prime}$ mass of $2.13 \mathrm{GeV}$. The string tension of $a=1.0 \mathrm{GeV} / \mathrm{fm}$ we used as our standard value in the flux tube model may be too large $(0.9 \mathrm{GeV} / \mathrm{fm}$ is more conventional), and the radial excitations are more sensitive to this difference than the other $q \bar{q}$ states, which we found to be in good agreement with experiment given $1.0 \mathrm{GeV} / \mathrm{fm}$. If we reduce the string tension in the $N=1 \mathrm{flux}$ tube model to the rather low value of $0.8 \mathrm{GeV} / \mathrm{fm}$, we find a mass of $M\left(D^{\prime}\right)=2.1 \mathrm{GeV}$, similar to Godfrey and Isgur. However the D-wave mass is then unacceptably low, $M(D)=1.54 \mathrm{GeV}$. The actual $D^{\prime} 2^{-+} q \bar{q}$ state is presumably near $2.2 \mathrm{GeV}$, with an uncertainty of at most about $0.1 \mathrm{GeV}$.

[39] F.E.Close, in Proceedings of the 3rd Workshop on the Tau Charm Factory (Edition Frontieres 1994), eds. J.Kirkby and R.Kirkby p.73; T.Barnes, ibid., p.41.

[40] Particle Data Group, Phys. Rev. D50, 1177 (1994).

[41] A.B.Clegg and A.Donnachie, Z. Phys C42, 455 (1994).

[42] A.Donnachie and Yu. Kalashnikova, Z.Phys C59, 621 (1993); A.Donnachie, Yu. Kalashnikova 
and A.Clegg, Manchester University Report MC-TH-93/10.

[43] G. Busetto and L. Oliver, Z. Phys.C 20, 247 (1983).

[44] R. Kokoski and N. Isgur, Phys. Rev. D35, 907 (1987).

FIG. 1. Ground state and first hybrid adiabatic potentials and their difference, for $N=1$. Solid lines are exact and dashed lines are the small oscillation approximation. String tension $a=1.0 \mathrm{GeV} / \mathrm{fm}$, bead mass $m_{b}=0.2 \mathrm{GeV}$.

FIG. 2. Hybrid potential gap $E_{1}(R)-E_{0}(R)$ for $N=1$ and $N=2$. Plotting conventions and parameters as in Fig.1; points are Monte Carlo.

FIG. 3. An $N=1$ quark, antiquark and flux-tube bead, showing the $q \bar{q}$-axis angles $\theta$ and $\phi$ and the rigid-body rotation angle $\phi_{b}$ relative to the reference configuration.

FIG. 4. Energies of the lightest $L=1,2 q \bar{q}$ and ${ }_{\Lambda} L={ }_{1} P$ hybrid states relative to $E_{0}=E_{S}$ for $N=1$. Lines show the adiabatic approximation and the points are Monte Carlo, $M=0$ (open) and $M=L$ (plus). Parameters $m_{b}=0.2 \mathrm{GeV}, a=1.0 \mathrm{GeV} / \mathrm{fm}, \alpha_{s}=0$.

FIG. 5. The lightest $L=0-3 q \bar{q}(q=u, d)$ and ${ }_{\Lambda} L={ }_{1} P,{ }_{1} D$ and ${ }_{2} D$ hybrid masses from Monte Carlo with physical parameters, $m_{q}=0.33 \mathrm{GeV}, m_{b}=0.2 \mathrm{GeV}, a=1.0 \mathrm{GeV} / \mathrm{fm}, \alpha_{s}^{f t}=1.3$. Square brackets denote masses used as input.

FIG. 6. Charmonium $c \bar{c}$ and hybrid masses, legend as in Fig.5. Parameters modified for charmonium are $m_{c}=1.5 \mathrm{GeV}$ and $\alpha_{s}^{f t}=0.72$. 
This figure "fig1-1.png" is available in "png" format from: http://arxiv.org/ps/hep-ph/9501405v2 
This figure "fig2-1.png" is available in "png" format from: http://arxiv.org/ps/hep-ph/9501405v2 
This figure "fig1-2.png" is available in "png" format from: http://arxiv.org/ps/hep-ph/9501405v2 
This figure "fig2-2.png" is available in "png" format from: http://arxiv.org/ps/hep-ph/9501405v2 
This figure "fig1-3.png" is available in "png" format from: http://arxiv.org/ps/hep-ph/9501405v2 
This figure "fig2-3.png" is available in "png" format from: http://arxiv.org/ps/hep-ph/9501405v2 